\title{
Grain-boundary relaxation and its effect on plasticity in nanocrystalline Fe
}

\author{
D. Jang \\ Department of Materials Science and Engineering, University of Michigan, Ann Arbor, Michigan 48109 \\ M. Atzmon ${ }^{\text {a) }}$ \\ Department of Nuclear Engineering and Radiological Sciences, University of Michigan, Ann Arbor, \\ Michigan 48109 and Department of Materials Science and Engineering, University of Michigan, \\ Ann Arbor, Michigan 48109
}

(Received 22 September 2005; accepted 6 February 2006; published online 20 April 2006)

\begin{abstract}
Grain-boundary relaxation in nanocrystalline Fe was studied by high-resolution transmission electron microscopy (HREM), and its effect on plasticity was characterized by nanoindentation. Samples with grain size of $9.8 \mathrm{~nm}$ were synthesized by mechanical attrition and subsequently annealed at low temperatures $\left(80\right.$ and $\left.100{ }^{\circ} \mathrm{C}\right)$ without affecting the grain size. While the hardness is not significantly affected by annealing, the strain-rate sensitivity peaks as a function of annealing time, suggesting two competing processes. HREM images show grain-boundary relaxation during annealing. Initially disconnected lattice planes were observed to evolve into a more continuous and ordered structure with regularly spaced grain-boundary dislocations. (c) 2006 American Institute of Physics. [DOI: 10.1063/1.2187417]
\end{abstract}

\section{INTRODUCTION}

Nanocrystalline materials, defined as polycrystals with grain size of less than a few tens of nanometers, were reported by Birringer et al., ${ }^{1}$ and have been attracting theoretical and practical interests due to their unique properties. For example, contrary to the typical strengthening of conventional coarse-grained materials with decreasing grain size, ${ }^{2,3}$ when the grain size is in the range of a few tens to a few nanometers, the dislocation pileup mechanism for Hall-Petch strengthening breaks down. ${ }^{4,5}$ Softening with decreasing grain size is observed, termed as inverse Hall-Petch behavior. ${ }^{6,7}$ For a grain size in the range of a few nanometers, the grain boundaries can occupy as much as $30 \%$ of the volume. ${ }^{8}$ Therefore, understanding the structure and behavior of grain boundaries is of essential importance to the study of nanocrystalline materials.

Many researchers have attempted to determine the structure of nanocrystalline materials. In studies of nanocrystalline Pd, synthesized by inert gas condensation, by highresolution transmission electron microscopy (HREM), Thomas et al. ${ }^{9}$ and Straub et al. ${ }^{10}$ reported that the lattice fringes from adjacent grains approached each other very closely at the grain boundary (at most $0.4 \mathrm{~nm}$ ), and that they changed direction abruptly at the boundary. This observation is consistent with molecular dynamics (MD) simulation result by Van Swygenhoven et al. ${ }^{11}$ who reported structural coherency at the grain boundary of nanocrystalline $\mathrm{Ni}$ and $\mathrm{Cu}$, created by Voronoi construction. ${ }^{12}$ However, different grain-boundary structure has also been reported. Interpreting X-ray diffraction data, Zhu et al. ${ }^{13}$ and Haubold et al. ${ }^{14}$ suggested an amorphous-layer-like grain-boundary structure for nanocrystalline $\mathrm{Fe},{ }^{13} \mathrm{Cu}$, and $\mathrm{Pd},{ }^{14}$ formed by inert gas condensation. MD simulation by Keblinski et al. ${ }^{15}$ also showed

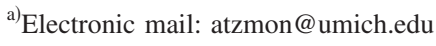

a "gluelike intergranular phase" for nanocrystalline Pd, formed by crystal growth from seeds embedded into a melt.

The fact that reports for nominally the same material are in conflict with each other suggests that the synthesis method or thermal history may affect the grain-boundary structure. Modification of the grain-boundary structure by heat treatment has been reported. Using X-ray atomic distribution function analysis, Löffler and Weissmüller ${ }^{16}$ reported that in nanocrystalline $\mathrm{Pd}$, formed by inert gas condensation, the disorder at or near the grain boundary decreases during annealing or aging. Using in situ $\mathrm{x}$-ray analysis, Moelle and Fecht ${ }^{17}$ observed that in ball-milled nanocrystalline $\mathrm{Fe}$, the lattice strain decreased significantly by annealing below $200{ }^{\circ} \mathrm{C}$ while the grain size remained constant. They suggested that this observation was likely due to structural relaxation of the grain boundaries. Grain-boundary relaxation by annealing was also observed by Hasnaoui et al. ${ }^{18}$ in MD simulation. They reported densification of the crystallographic planes of each grain at grain boundary by annealing at $800 \mathrm{~K}$ for $100 \mathrm{ps}$. Using HREM for artificially formed grain boundaries of bicrystals, Merkle ${ }^{19}$ has performed extensive research on grain-boundary relaxation in metals and ceramics, classifying grain-boundary relaxation into four different modes.

Given that grain-boundary activity contributes significantly to plasticity of nanocrystalline materials, ${ }^{20,21}$ their mechanical behavior is likely to be affected by grain-boundary relaxation. The strain-rate sensitivity, defined as $m$ $=d \ln \sigma / d \ln \dot{\varepsilon}$, where $\sigma$ is the applied stress and $\dot{\varepsilon}$ is the corresponding strain rate, is a useful parameter for understanding the grain-boundary contribution to plastic deformation because grain-boundary activity is thermally activated, and thus strain-rate dependent. ${ }^{21}$ We have previously reported ${ }^{7}$ that the strain-rate sensitivity increases monotonically as the grain size decreases (Fig. 1). This observation suggests that increased grain-boundary volume fraction at smaller grain size increases the contribution of grain- 


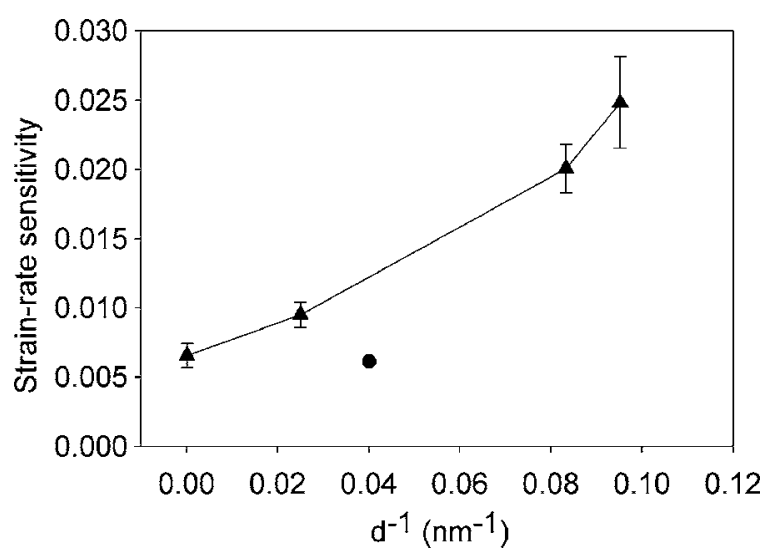

FIG. 1. Strain-rate sensitivity as a function of inverse grain size. The solid circle (O) represents the value for Fe with $24 \mathrm{~nm}$ grains, which had experienced heat treatment, therefore, grain growth and grain-boundary relaxation (Ref. 22).

boundary activity to deformation, making the stress more sensitive to the strain rate. Also, the strain-rate sensitivity of sintered nanocrystalline $\mathrm{Fe}^{22}$ which had undergone grain growth, was lower than that of an as-milled sample ${ }^{7}$ with the same grain size (see Fig. 1), suggesting that grain-boundary relaxation, caused by heat treatment, possibly affected grainboundary plasticity.

In the present study, relaxation of grain boundaries in nanocrystalline Fe and its effect on plasticity are investigated by differential scanning calorimetry (DSC), nanoindentation, and HREM. Nanocrystalline Fe samples with volumeaveraged grain size of $9.8 \mathrm{~nm}$ were synthesized by ball milling and then annealed at low temperatures that did not affect the grain size. The effect of annealing on plasticity was studied by nanoindentation. The atomic structure of grain boundaries and its dependence on annealing time were observed using HREM.

\section{EXPERIMENTAL PROCEDURE}

Fe powder of $99.9 \%$ purity was obtained from Cerac Company, with all samples originating from the same batch. Nanocrystalline $\mathrm{Fe}$ was prepared at room temperature by high-energy ball milling in Ar in a Spex 8000 Mixer/Mill, using a stainless steel vial and balls, for $24 \mathrm{~h}$. The ball-tosample weight ratio for high-energy ball milling was about $4: 1$. For comparison, one sample was prepared by lowenergy ball milling in Ar using a Fritsch Pulverisette-0 vibrating-frame ball mill for $100 \mathrm{~h}$. In this case, $3.2 \mathrm{~g}$ of Fe powder were milled with a stainless steel ball of $5 \mathrm{~cm}$ diameter and $500 \mathrm{~g}$ weight. Unless otherwise noted, results are quoted for samples formed by high-energy ball milling. We have previously ruled out a significant effect of impurities acquired during ball milling on subsequent mechanical behavior. ${ }^{7}$ Samples were annealed at 80 and $100{ }^{\circ} \mathrm{C}$ from $30 \mathrm{~min}$ to $24 \mathrm{~h}$ in a Ti-gettered flowing-Ar atmosphere or in vacuum $\left(<10^{-8}\right.$ torr $)$.

$\mathrm{X}$-ray diffraction (XRD) was performed using a horizontal General Electric $\theta-2 \theta$ powder diffractometer in stepscanning mode with Mo $K \alpha$ radiation $(\lambda=0.07093 \mathrm{~nm})$. The peaks were fitted with a Pearson VII function, and the volume-averaged grain size and root mean square (rms) stain were determined by the Warren-Averbach analysis. ${ }^{23}$ To prepare samples for hardness and strain-rate sensitivity measurements, the powder was consolidated under $17 \mathrm{MPa}$ at room temperature and then cold mounted with epoxy resin. The powder particles were at least $15 \mu \mathrm{m}$ in diameter. The hardness and strain-rate sensitivity were measured using Nanoinstruments NANO INDENTER $®$ II with a Berkovich tip. Measurements were conducted in displacement-control mode at a rate of $10 \mathrm{~nm} / \mathrm{s}$ to a maximum depth of $300 \mathrm{~nm}$. Thus, the indent was significantly smaller than the particle, but significantly larger than the grain size. The absence of surface cracks due to indentation was confirmed by scanning electron microscopy (SEM). To determine the strain-rate sensitivity, the indenter tip was kept at constant load, $20 \mathrm{mN}$, and the displacement of the tip was monitored as a function of time. ${ }^{7}$ Each reported value of hardness or strain-rate sensitivity was obtained by averaging 25-30 measured values. The standard deviation of the mean was indicated as an estimate of error due to experimental scatter. DSC measurements were performed with a Perkin-Elmer DSC 7. Approximately $40 \mathrm{mg}$ of nanocrystalline Fe powder were sealed in $\mathrm{Al}$ pans and scanned at a heating rate of $20 \mathrm{~K} / \mathrm{min}$ to the maximum temperature of $750 \mathrm{~K}$. To determine the baseline, a second run was conducted without interruption, using the same scanning conditions, and its trace was subtracted from that of the first run to obtain the intrinsic heat evolution of the sample. For each sample condition, curves from two individual runs were averaged.

Samples were observed using a JEOL 3011 HREM. The grain-boundary structure is only visible by HREM when the specimen thickness is similar to the grain size and when the grain boundary is aligned edge-on. To prepare sufficiently thin nanocrystalline Fe specimen $(\sim 10 \mathrm{~nm})$, the powder particles were embedded into a $\mathrm{Cu}$ foil. The resulting composite was chemically jet thinned from the $\mathrm{Cu}$ side until it was perforated, using an electrolyte consisting of $10 \%$ perchloric acid and $90 \%$ methanol at $-50{ }^{\circ} \mathrm{C}$, with an applied voltage of $\sim 10 \mathrm{~V}$ and current density of $\sim 7 \mathrm{~mA} / \mathrm{mm}^{2}$, respectively. The thinnest portion, near the perforation, was used for HREM. To avoid possible structural damage to the samples, mechanical grinding or ion milling was avoided.

\section{RESULTS}

The average grain size of the as-milled samples, determined by $\mathrm{x}$-ray diffraction, ${ }^{23}$ was $9.8 \mathrm{~nm}$. This agrees with our HREM observation. In Fig. 2, the grain size and rms strain of samples annealed in the Ti-gettered flowing-Ar furnace at 80 and $100{ }^{\circ} \mathrm{C}$ are displayed as a function of annealing time. The rms strain decreases slightly at the early stage of the annealing and then remains nearly unchanged. The grain size remains nearly constant for the entire range of annealing time. In order to observe changes in enthalpy content upon annealing, two samples were investigated by DSC: as-milled nanocrystalline $\mathrm{Fe}$ and a milled sample, subsequently annealed at $100{ }^{\circ} \mathrm{C}$ for $24 \mathrm{~h}$ in vacuum. In Fig. 3, the DSC traces, each obtained by averaging two curves, measured at a heating rate of $20 \mathrm{~K} / \mathrm{min}$, are displayed. The as- 

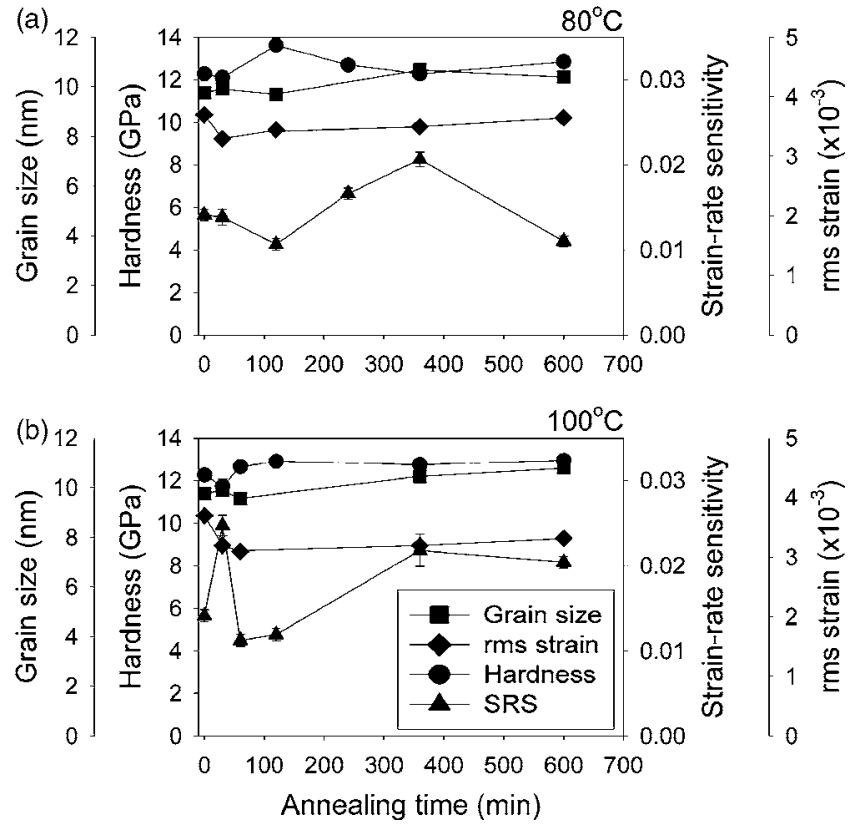

FIG. 2. Grain size, rms strain, hardness, and strain-rate sensitivity as functions of annealing time at (a) $80{ }^{\circ} \mathrm{C}$ and (b) $100{ }^{\circ} \mathrm{C}$. The samples were annealed in Ti-gettered Ar.

milled sample shows an exothermic peak starting at around $370 \mathrm{~K}$, but the annealed sample does not show a significant signal up to around $400 \mathrm{~K}$. A shoulder, observed on the exothermic peak of the as-milled sample around $445 \mathrm{~K}$ (arrow in Fig. 3), is not present for the annealed sample. The enthalpy releases of as-milled and annealed nanocrystalline $\mathrm{Fe}$ in the range of 323-552 $\mathrm{K}$ (between two dashed lines in Fig. 3) were 950 and $630 \mathrm{~J} / \mathrm{mol}$, respectively. Thus, annealing leads to a decrease of $320 \mathrm{~J} / \mathrm{mol}( \pm 20 \%)$ in stored enthalpy.

The hardness and strain-rate sensitivity of the nanocrystalline Fe samples, which were annealed at 80 and $100{ }^{\circ} \mathrm{C}$ for various times, were measured by nanoindentation. The results are displayed in Fig. 2, together with grain size and rms strain, as a function of annealing time under Ti-gettered Ar. The hardness varies just slightly with annealing time. The initial strain-rate sensitivity of the as-milled sample, 0.012, increases above 0.2 after $6 \mathrm{~h}$ for both temperatures. At $80{ }^{\circ} \mathrm{C}$, the strain-rate sensitivity decreases slightly below the initial value after $10 \mathrm{~h}$, but at $100{ }^{\circ} \mathrm{C}$, it is still higher than

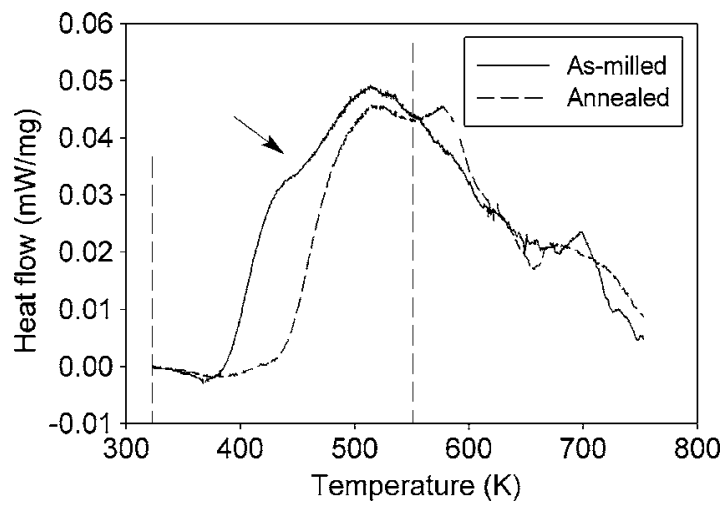

FIG. 3. DSC traces for as-milled and annealed $\left(24 \mathrm{~h}\right.$ at $\left.100{ }^{\circ} \mathrm{C}\right)$ nanocrystalline $\mathrm{Fe}$

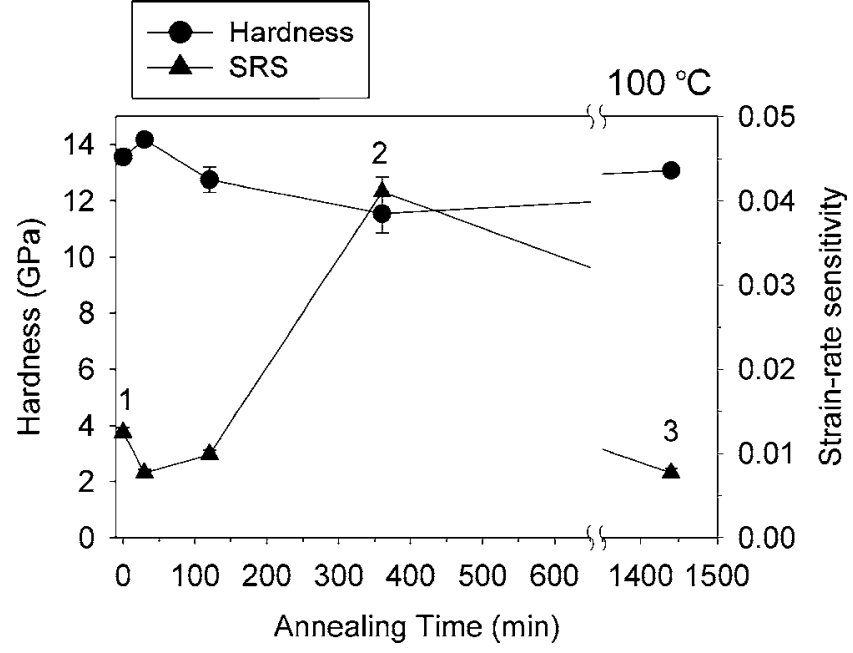

FIG. 4. Hardness and strain-rate sensitivity as functions of annealing time for samples annealed in vacuum at $100{ }^{\circ} \mathrm{C}$.

the initial value after $10 \mathrm{~h}$. Furthermore, at $100{ }^{\circ} \mathrm{C}$, a narrow peak is observed at $30 \mathrm{~min}$. A similar narrow peak was also observed in samples that were formed by low-energy ball milling and subsequently annealed at $100{ }^{\circ} \mathrm{C}$ for up to $6 \mathrm{~h}$ under Ti-gettered Ar. It is noted that the hardness variations follow closely those of the strain-rate sensitivity, but in the opposite direction. The same measurements were also performed on samples annealed in vacuum at $100{ }^{\circ} \mathrm{C}$, as shown in Fig. 4. The initial strain-rate sensitivity of 0.012 increases above 0.04 after $6 \mathrm{~h}$ and then decreases slightly below the initial value after $24 \mathrm{~h}$. However, the strain-rate sensitivity of samples annealed in vacuum does not peak at $30 \mathrm{~min}$, as it does for the sample annealed under Ti-gettered Ar at the same temperature.

Changes in atomic configuration at or near grain boundaries during annealing were observed using HREM. Three samples were selected for observation: as-milled, annealed $6 \mathrm{~h}$ at $100{ }^{\circ} \mathrm{C}$, which has the highest strain-rate sensitivity value, and annealed $24 \mathrm{~h}$ at $100{ }^{\circ} \mathrm{C}$, both in vacuum. These are labeled as 1, 2, and 3, respectively, in Fig. 4. 16, 4, and 12 grain boundaries were imaged for each of the three samples, respectively. In Figs. 5 and 6, typical grainboundary images are shown for an as-milled (1 in Fig. 4) and annealed ( $100{ }^{\circ} \mathrm{C} 24$ h, 3 in Fig. 4) samples, respectively. In both Figs. 5 and 6 , grain $I$ is aligned along the [111] zone axis, and the three lattice fringes observed correspond to the (101), (110), and (01) planes. The interplanar spacing in grain II is within $3 \%$ of the values in grain I, allowing us to identify the planes observed in grain II as (110). It should be noted that the instrumental line-to-line resolution, $0.14 \mathrm{~nm}$, is close to the (200) interplanar spacing, $0.143 \mathrm{~nm}$, allowing only (110) planes to be clearly resolved. This is the reason that no other lattice fringes are observed in any of the images. The angles between the (110) planes in neighboring grains are about $21^{\circ}$ in both Figs. 5 and 6 . It should be noted that, because of the rotational degree of freedom about the [110] axis in grain II, a determination of the misorientation angle between the grains is not possible. However, considering all (110) planes in grain II, the angle observed between 


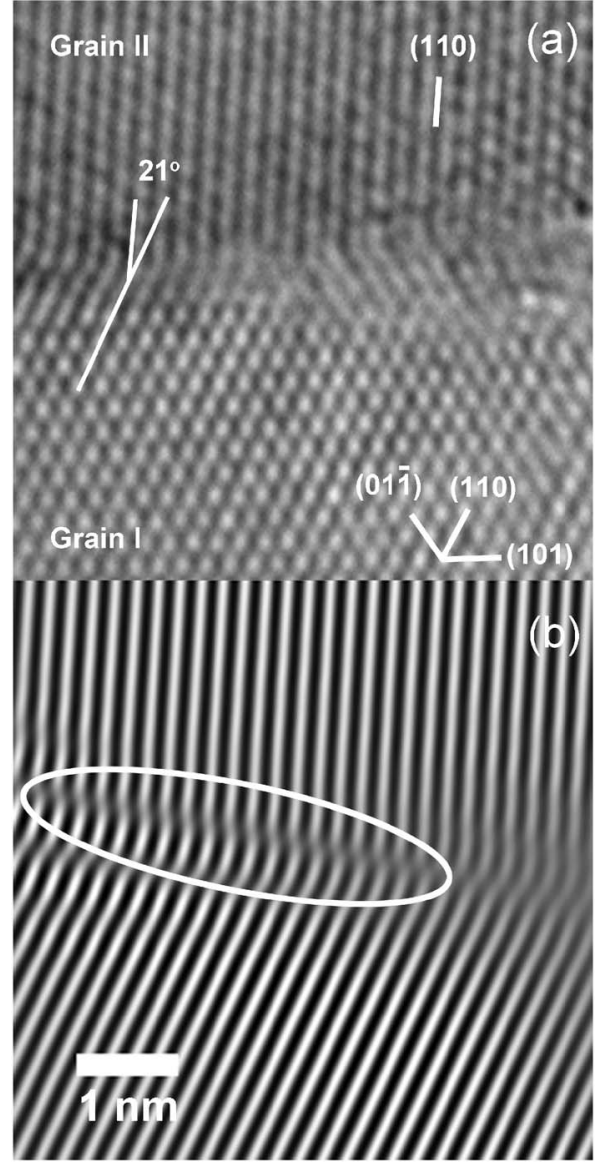

FIG. 5. (a) HREM image of a grain boundary in as-milled nanocrystalline Fe. Grain $I$ is aligned along the $[\overline{1} 11]$ zone axis. The angle between the (110) planes in grain I and grain II is $21^{\circ}$. (b) Fourier-filtered image. Disconnected lattice fringes can be observed at the grain boundary (encircled).

the planes is a lower limit on the misorientation angle between the grains. Thus, the boundaries in Figs. 5 and 6 are high-angle grain boundaries.

To highlight the atomic structure at and near the grain boundary, the images were Fourier filtered, so that only one set of lattice fringes in each grain, aligned approximately perpendicular to the grain boundary, is visible [Figs. 5(b) and 6(b)]. The clearest difference between samples was found in the connectivity of lattice fringes at the grain boundary. In as-milled $\mathrm{Fe}$, all lattice fringes are disconnected at the grain boundary, where a gap is observed [Fig. 5(b), encircled]. However, in the sample annealed at $100{ }^{\circ} \mathrm{C}$ for $24 \mathrm{~h}$, a significant portion of the lattice fringes is continuous, and the misfit of lattice planes is accommodated by regularly spaced grain-boundary dislocations [Fig. 6(b)]. Attempts to observe samples in situ in the HREM during heating were not successful-samples curled up instantly upon heating. Furthermore, even if such attempts were successful, the observed behavior could be severely affected by surface effects. We have confidence in our results because of their reproducibility. The observations we report above are typical- 15 of 16 and 12 of 12 grains boundaries, respectively, had the same structure. For each of the two sample types, one to two of the boundaries observed were possibly low-angle boundaries $\left[<15^{\circ}\right.$ between the (110) planes], and even these followed

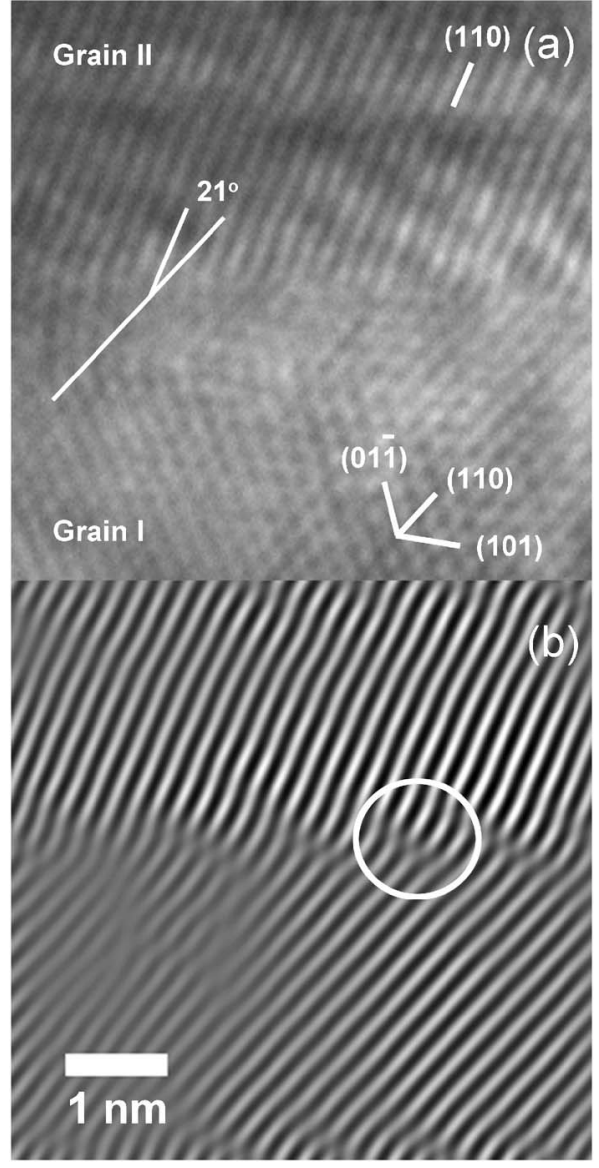

FIG. 6. (a) HREM image of a grain boundary in annealed nanocrystalline $\mathrm{Fe}$ at $100{ }^{\circ} \mathrm{C}$ for $24 \mathrm{~h}$. Grain I is aligned along the [111] zone axis. The angle between the (110) planes in grain I and grain II is $21^{\circ}$. (b) Fourier-filtered image. Regularly spaced grain-boundary dislocations (encircled) are observed at the grain boundary.

the same trends as the high-angle boundaries. In Figs. 7-9, grain-boundary images of a sample annealed for $6 \mathrm{~h}$ at $100{ }^{\circ} \mathrm{C}$ are displayed. Unlike samples 1 and 3, whose grainboundary structures are nearly uniform, the $100{ }^{\circ} \mathrm{C} 6 \mathrm{~h}$ annealed sample contains a mixture of the grain-boundary structures observed in Figs. 5 and 6: some grain boundaries have a structure typical of the as-milled sample (Fig. 7), and others have a structure typical of the $100{ }^{\circ} \mathrm{C} 24 \mathrm{~h}$ annealed sample (Fig. 8). Furthermore, in some boundaries, both structures are observed side by side in a single grain boundary (Fig. 9), suggesting that the grain-boundaries are in the middle of a transition between the initial and steady-state structures. It is finally noted that no lattice dislocations were found in any of the samples observed.

\section{DISCUSSION}

The following macroscopic observations suggest relaxation of the grain boundaries: (1) the decrease in rms strain at fixed grain size, (2) the shift in onset temperature of the exothermic peak in DSC trace from $\sim 370$ to $\sim 400 \mathrm{~K}$ (Fig. 3 ), and (3) the decrease in stored enthalpy by $320 \mathrm{~J} / \mathrm{mol}$. Since the material does not contain lattice dislocations, ${ }^{24}$ the observed changes cannot be attributed to dislocations pro- 


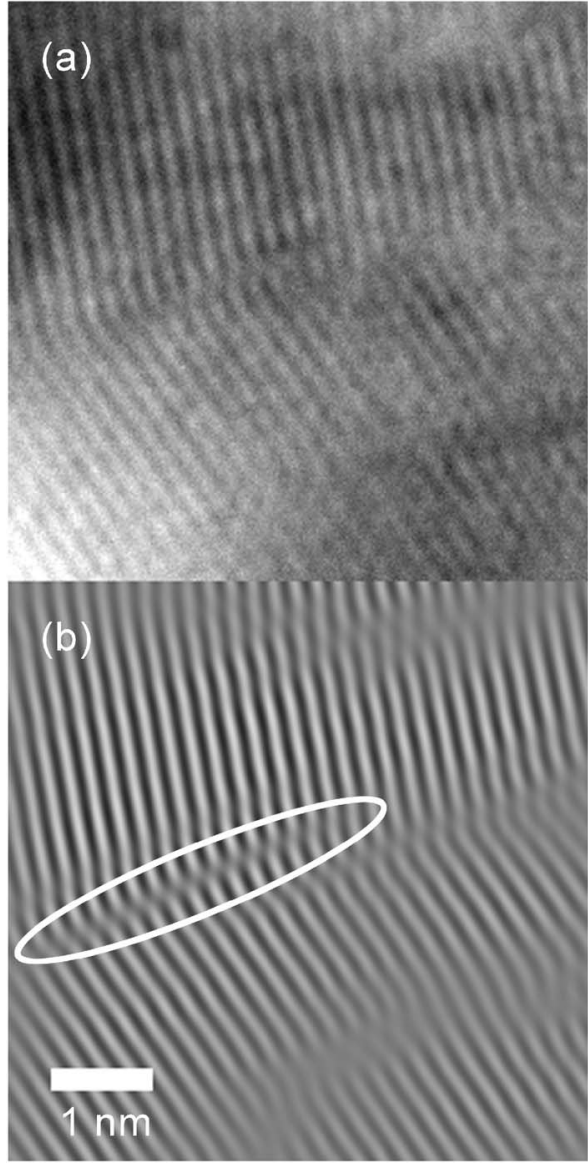

FIG. 7. (a) HREM image of a grain boundary in $100{ }^{\circ} \mathrm{C} 6 \mathrm{~h}$ annealed nanocrystalline Fe. (b) Fourier-filtered image. It has a structure similar to that of grain boundaries in as-milled nanocrystalline Fe.

cesses, such as polygonization. Consequently, it is plausible to attribute the relaxation to the grain boundaries.

Assuming the grain shape to be the regular 14-sided tetrakaidecahedron, and the grain size to be the maximum diameter of an inscribed sphere, ${ }^{8}$ the grain-boundary area per unit volume is $3.44 \times 10^{8} \mathrm{~m}^{-1}$ for an average grain diameter of $9.8 \mathrm{~nm}$. Assuming the decrease in stored enthalpy, $320 \mathrm{~J} / \mathrm{mol}$, is due to grain-boundary relaxation only, it corresponds to a decrease of grain-boundary enthalpy by $0.13 \mathrm{~J} / \mathrm{m}^{2}$. The average grain-boundary free energy of bcc $\mathrm{Fe}$ is about $1.96 \mathrm{~J} / \mathrm{m}^{2},{ }^{25}$ thus the energy decrease is about $7 \%$ of the total grain-boundary free energy. Moelle and Fecht ${ }^{17}$ report for as-milled Fe powder a DSC curve that is roughly similar to ours, if one accounts for the differences in grain size and heating rates. Using the total enthalpy release due to extensive grain growth, and comparing it with theoretically calculated grain-boundary energy values, they estimate a higher contribution of relaxation to the stored enthalpy than our value. While it is possible that our annealed sample is not fully relaxed, we note that our measurement provides a more direct estimate of the enthalpy associated with relaxation. In addition, grain diameters were determined in Ref. 17 using the integral breadths of Bragg peaks, which yield results that are different from those of the WarrenAverbach method. ${ }^{26}$

The following pitfalls should be noted when interpreting

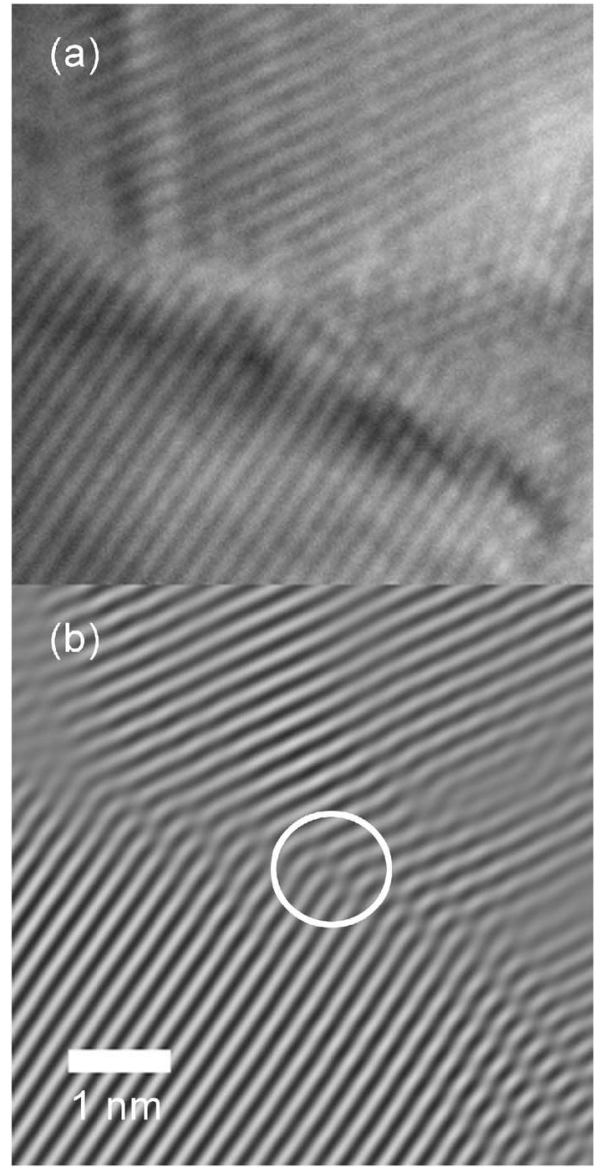

FIG. 8. (a) HREM image of a grain boundary in $100{ }^{\circ} \mathrm{C} 6 \mathrm{~h}$ annealed nanocrystalline Fe. (b) Fourier-filtered image. It has a structure similar to that of grain boundaries in $100{ }^{\circ} \mathrm{C} 24 \mathrm{~h}$ annealed nanocrystalline $\mathrm{Fe}$.

HREM images: (1) Attempts at in situ annealing in the HREM were not successful. The differences in the grainboundary structures are determined only statistically based on ex situ observation. (2) The HREM images of nanocrystalline samples may contain artifacts such as overlap or grain boundaries that are not aligned edge-on. (3) The Fourier filtering technique, which was used to highlight the lattice fringes, may induce an artifact because the Fourier transform of a finite domain has finite-size ripples in reciprocal space. Nonetheless, the conclusion on the evolution of the grainboundary structure is still sound because of its statistical consistency.

The grain-boundary structure is known to depend on the boundary misorientation. ${ }^{27}$ Therefore, in order to study the effect of annealing on the grain-boundary structure, boundaries of similar misorientation should be compared for different samples. In the present study, two grain boundaries before and after annealing, displayed in Figs. 5 and 6, have the same lower bound on their misorientation angle, $21^{\circ}$, ruling out the possibility that the difference observed in the grain-boundary structure is due to one of them being a lowangle boundary. Furthermore, the authors observed a discontinuous grain-boundary structure in the as-milled sample for both high-angle grain boundaries and those that may be lowangle boundaries, but for neither boundary type in the sample annealed for $24 \mathrm{~h}$. Because low-angle grain bound- 


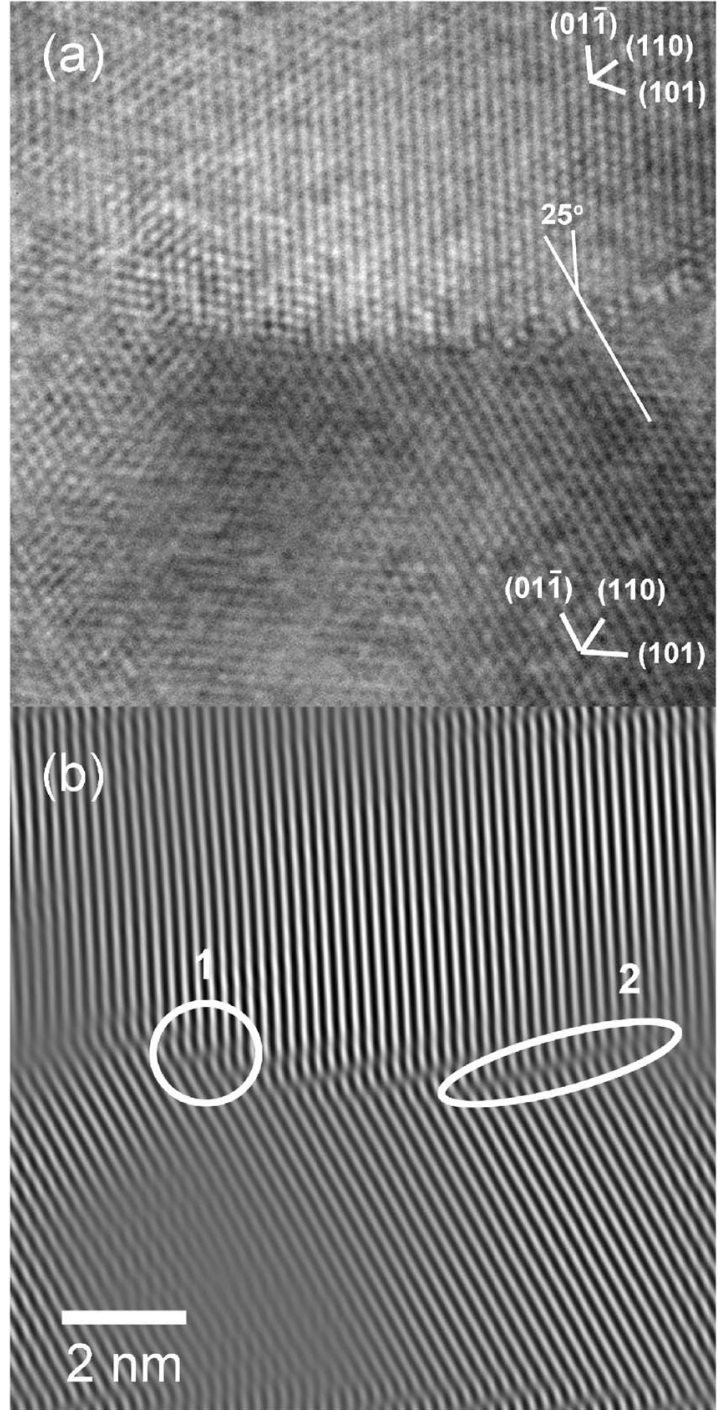

FIG. 9. (a) HREM image of a grain boundary in $100{ }^{\circ} \mathrm{C} 6 \mathrm{~h}$ annealed nanocrystalline Fe. (b) Fourier-filtered image. A single grain boundary exhibits both continuous (1) and discontinuous (2) lattice fringes.

aries generally have a more-ordered structure, ${ }^{27}$ this observation strengthens the conclusion that the as-milled sample has a discontinuous grain-boundary structure, which evolves into a more-ordered structure due to annealing.

The HREM images suggest that at $100{ }^{\circ} \mathrm{C}$, the initially discontinuous grain-boundary structure is transformed into a more-ordered structure with regularly spaced grain-boundary dislocations. The latter structure likely corresponds to a relaxed state with lower free energy. Relaxation is likely to lead to strengthening ${ }^{18}$ and to a decrease of the strain-rate sensitivity. For all isothermal annealing conditions, contrary to expectation, the strain-rate sensitivity does not evolve monotonically, but goes through a peak (Figs. 2 and 4). This suggests that there is not a single process responsible for its evolution, but that at least two processes compete. Further study is required to understand which additional process may be responsible for the initial increase of the strain-rate sensitivity. Considering the effect of annealing atmosphere, we cannot rule out a role of impurities in the evolution of the strain-rate sensitivity.

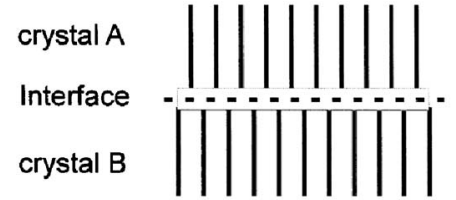

(a) $\mathrm{p}=0$, no dislocations

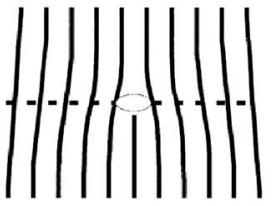

(c) $\mathrm{p}=1$, localized core

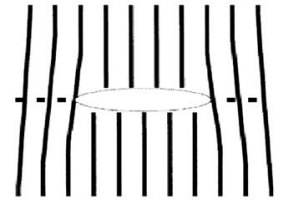

(b) $\mathrm{p}=\mathbf{0 . 3}$, delocalized core

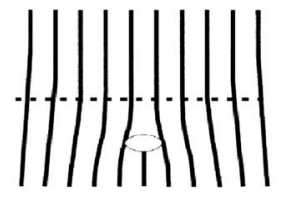

(d)
FIG. 10. The degree of matching across the grain boundary as a function of $p$ value [after Lojkowski and Fecht (Ref. 28)]. The lattice connectivity increases from (a) to (c). $P>1$ is unlikely for an elemental solid.

In the presence of grain-boundary dislocations, as we report, one set of parameters describing the grain boundary are the characteristics of the dislocations, e.g., dislocation core width. The relation between the grain-boundary structure and the grain-boundary dislocation distribution was analyzed by Lojkowski and Fecht, ${ }^{28}$ who introduced the concept of the localization parameter, $p$. It is defined as the ratio of core widths of lattice and grain-boundary dislocations or, equivalently, as the ratio of shear modulus of the grain interior and grain boundary, and provides a criterion for classifying the type of grain boundary. In Fig. 10, grain-boundary types are illustrated for different values of $p$.

According to Lojkowski and Fecht, ${ }^{28}$ given that the localization parameter is equal to the ratio of the shear modulus of the grain interior and grain boundary, the transition of the grain boundary to a thermodynamically more-stable state can induce a grain-boundary structure change via a change in the grain-boundary shear modulus. That is, if a grain boundary relaxes by a thermal process, the increased bond strength of atoms at the grain boundary can induce an increase of lattice plane connectivity via an increase of the grainboundary shear modulus. Observation of the grain-boundary structure of nanocrystalline $\mathrm{Fe}$ in the present study suggests that the transformation described above takes place during low-temperature annealing. The initially discontinuous lattice planes of as-milled $\mathrm{Fe}$ become continuous, with regularly spaced grain-boundary dislocations, as a result of annealing. The decreasing portion of the strain-rate sensitivity curve (Figs. 2 and 4 ) is possibly due to the stiffening of grain boundaries and their enhanced connectivity.

\section{SUMMARY}

Grain-boundary relaxation and its effect on the plasticity of nanocrystalline Fe were studied using DSC, nanoindentation, and HREM. Nanocrystalline Fe samples with identical grain size, but different degrees of grain-boundary relaxation, were obtained by high-energy ball milling and subsequent annealing at low temperatures $\left(80\right.$ and $\left.100{ }^{\circ} \mathrm{C}\right)$. While the hardness was not affected significantly by annealing, the strain-rate sensitivity shows a peak value at an intermediate annealing time and decreases to slightly below its initial value, suggesting two competing processes. HREM images 
suggest that grain boundaries evolve gradually from an initially discontinuous structure to a more continuous and ordered structure with regularly spaced grain-boundary dislocations. An artifact due to a bias in grain-boundary misorientation is shown to be unlikely. This relaxation process is suggested to be responsible for a decrease of strainrate sensitivity by annealing. However, in order to closely relate the evolution of structure with that of the strain-rate sensitivity, further study is required.

\section{ACKNOWLEDGMENTS}

The authors acknowledge useful discussions with Professor A. Ghosh and Professor L. M. Wang. This work was funded by the National Science Foundation, Grant No. DMR-0314214.

${ }^{1}$ R. Birringer, H. Gleiter, H.-P. Klein, and P. Marquardt, Phys. Lett. 102A, 365 (1984).

${ }^{2}$ E. O. Hall, Proc. Phys. Soc. London, Sect. B 64, 747 (1951).

${ }^{3}$ N. J. Petch, J. Iron Steel Inst., London 174, 25 (1953).

${ }^{4}$ V. G. Gryaznov, V. A. Solovev, and L. I. Trusov, Scr. Metall. Mater. 24, 1529 (1990).

${ }^{5}$ T. G. Nieh and J. Wadsworth, Scr. Metall. Mater. 25, 955 (1991).

${ }^{6}$ C. C. Koch and J. Narayan, Mater. Res. Soc. Symp. Proc. 634, B5.1.1 (2001).

${ }^{7}$ D. Jang and M. Atzmon, J. Appl. Phys. 93, 9282 (2003).

${ }^{8}$ G. Palumbo, S. J. Thorpe, and K. T. Aust, Scr. Metall. Mater. 24, 1347 (1991).
${ }^{9}$ G. J. Thomas, R. W. Siegel, and J. A. Eastman, Scr. Metall. Mater. 24, 201 (1990).

${ }^{10}$ W. M. Straub, T. Gessmann, W. Sigle, F. Phillipp, A. Seeger, and H. E. Schaefer, Nanostruct. Mater. 6, 571 (1995).

${ }^{11}$ H. Van Swygenhoven, D. Farkas, and A. Caro, Phys. Rev. B 62, 831 (2000).

${ }^{12}$ G. Z. Voronoi, J. Reine Angew. Math. 134, 199 (1908).

${ }^{13}$ X. Zhu, R. Birringer, U. Herr, and H. Gleiter, Phys. Rev. B 35, 9085 (1987).

${ }^{14}$ T. Haubold, R. Birringer, B. Lengeler, and H. Gleiter, Phys. Lett. A $\mathbf{1 3 5}$, 461 (1989).

${ }^{15}$ P. Keblinski, D. Wolf, S. R. Phillpot, and H. Gleiter, Scr. Mater. 41, 613 (1999).

${ }^{16}$ J. Löffler and J. Weissmüller, Phys. Rev. B 52, 7076 (1995).

${ }^{17}$ C. H. Moelle and H.-J. Fecht, Nanostruct. Mater. 6, 421 (1995).

${ }^{18}$ A. Hasnaoui, H. Van Swygenhoven, and P. M. Derlet, Acta Mater. 50, 3927 (2002).

${ }^{19}$ K. L. Merkle, Microsc. Microanal. 3, 339 (1997).

${ }^{20}$ H. Conrad and J. Narayan, Scr. Mater. 42, 1025 (2000).

${ }^{21}$ H. Van Swygenhoven, M. Spaczer, and A. Caro, Acta Mater. 47, 3117 (1999).

${ }^{22}$ T. R. Malow, C. C. Koch, P. Q. Miraglia, and K. L. Murty, Mater. Sci. Eng., A 252, 36 (1998).

${ }^{23}$ B. E. Warren, X-ray Diffraction (Dover, New York, 1990), pp. 262-275.

${ }^{24}$ H. Van Swygenhoven, D. Farkas, and A. Caro, Phys. Rev. B 62, 831 (2000).

${ }^{25}$ A. Cottrell, An Introduction to Metallurgy, 2nd ed. (Edward Arnold Ltd., London, 1975), p. 339.

${ }^{26}$ H. H. Tian and M. Atzmon, Philos. Mag. A 79, 1769 (1999).

${ }^{27}$ D. Wolf and S. Yip, Materials Interfaces (Chapman and Hall, London, 1992).

${ }^{28}$ W. Lojkowski and H.-J. Fecht, Prog. Mater. Sci. 45, 339 (2000). 